\title{
Using Automated Agents to Teach Negotiation
}

\author{
Emmanuel Johnson \\ Institute for Creative Technologies \\ University of Southern California \\ 12015 E Waterfront Dr, Los Angeles, CA 90094
}

\begin{abstract}
Negotiation is an integral part of our daily lives regardless of occupation. Although ubiquitous to our experience, we are never taught to negotiate. This lack of training presents many consequences from unfair salary negotiation to geopolitical ramification. The ability to resolve conflicts and negotiate is becoming more critical due to the rise of automated systems which look to replace various repetitive task jobs. In hopes of improving human negotiation skills, my work seeks to develop automated negotiation agents capable of providing personalized feedback. In this paper, I provide an overview of my past , current, and future work.
\end{abstract}

\section{Background}

Negotiation is an integral part of our daily lives regardless of occupation. Although ubiquitous to our experience, we are never thought to negotiate. This lack of training presents both many challenges from unfair salary negotiation to geopolitical ramification. The ability to resolve conflicts and negotiate is becoming more critical due to the rise of automated systems which look to replace various repetitive task jobs. In a recent study, researchers projected a increase in need for jobs that require collaborative and negotiation skills. This would increase the need for individuals with softer skills and the ability to work collaboratively. To improve one's negotiation ability, a number of courses are offered through universities business, and law school. The issue with these courses is that they can be quite expensive.Automated agents provide a low cost alternative. Research has shown the benefits of using automated agents to teach negotiation (Gratch et al. 2015). Research shows that even by just allowing users to practice with an automated agents, people are able to improve their negotiation ability(Lin, Oshrat, and Kraus 2009). A number of systems have been proposed and develop to study how humans negotiate as well as help individuals improve their negotiation skills. Systems such as the conflict resolution agents(Gratch, DeVault, and Lucas 2016), pocket negotiators(Hindriks and Jonker 2008), IAGO (Mell and Gratch 2016) and Bilat (Kim et al. 2009) have all been shown as effective tools to allow humans to negotiate with human-like automated agents.

Copyright (C) 2019, Association for the Advancement of Artificial Intelligence (www.aaai.org). All rights reserved.
Experiential Learning Theory states that learning is best viewed as a process rather than an outcome. This process is enhanced when learners are able to engage their cognitive abilities, emotion, perception, and behavior (Kolb and Kolb 2012) and can reflect upon their subsequent actions. Thus, feedback plays a valuable role in the learning process. It informs students of their performance, and deficiencies. Thus for multi-agent systems to be effective teaching system, these systems must provide an opportunity for students to practice, review their performance and receive actionable feedback based on similar metrics those used in the class room.In the next section, I highlight my current work in this page followed by future work.

\section{Current Work}

In building a negotiation training system, we must first consider what aspect of negotiation we want to teach. Once we know what we want to teach, the next step is understanding the student. With a subject and understanding of our pupil, the goal is to provide actionable feedback that would help them improve. We began by first identifying a set of principles followed by good negotiators. These principles were proposed by Harold Kelley and has been subsequently validated by research (Kelley 1996). Kelley found that good negotiators avoided early concession, made efficient concessions and encouraged their opponent to concede. In a previously published paper(Johnson, Gratch, and DeVault 2017), I showed how Kelley's principles of a good negotiators could be translated into quantifiable metrics that are easily extracted via automated methods. This paper utilized a corpus of human-agent negotiation with the CRA agent. I also showed that these metrics were indeed predictive of negotiation outcome and could be used to provide feedback. My next set of studies were geared towards finding ways to teach individuals best practices by providing feedback based on these automated metrics. To better understand how these metrics help people improve their negotiation abilities, we conducted a study which looked at the effects of personalized feedback in helping to improve negotiation skills (Monahan et al. 2018). Participants negotiated with two agents and were either provided feedback after the first negotiation, or not. In this paper, we showed that individuals who were provided with personalized feedback did do better in a subsequent negotiation than those who did not receive feed- 
back. This showed that indeed our metrics are working and that individuals who received personalized feedback did get better. This study focused primarily on whether or not feedback matter and did not make a distinction between the type of feedback(generic vs personalized). We are able to show that participants are improving their value claiming (getting more value from the negotiation) but not their value creating strategy(understanding your opponent's preference so that a win-win solution can be found).

\section{Future Work}

Currently, we are able to show how one can design a pedagogical learning agent to teach negotiation. However, my work has focus primarily on teaching the more mechanistic aspect of a negotiation (offers, information sharing, questions asked, etc). We know that a negotiation involves more than just offers and statements being exchanged, and the subjective outcome matters. People care about the social element of a negotiation (Bazerman et al. 2000) as well as the fairness. The deal they receive should not disproportionately favors their opponent. In a follow up studies, I looked to examine subjective outcome of a negotiation and provide feedback to users on that. This would require included more subjective measures of a negotiation as part of the current metrics. I also hope to move from the Conflict Resolution Agent platform to IAGO.

\section{References}

Bazerman, M. H.; Curhan, J. R.; Moore, D. A.; and Valley, K. L. 2000. Negotiation. Annual Review of Psychology 51(1):279-314. PMID: 10751973.

Gratch, J.; DeVault, D.; Lucas, G. M.; and Marsella, S. 2015. Negotiation as a challenge problem for virtual humans. In Brinkman, W.-P.; Broekens, J.; and Heylen, D., eds., Intelligent Virtual Agents, 201-215. Cham: Springer International Publishing.

Gratch, J.; DeVault, D.; and Lucas, G. 2016. The benefits of virtual humans for teaching negotiation. In Traum, D.; Swartout, W.; Khooshabeh, P.; Kopp, S.; Scherer, S.; and Leuski, A., eds., Intelligent Virtual Agents, 283-294. Cham: Springer International Publishing.

Hindriks, K. V., and Jonker, C. M. 2008. Creating humanmachine synergy in negotiation support systems: Towards the pocket negotiator. In Proceedings of the 1st International Working Conference on Human Factors and Computational Models in Negotiation, 47-54. ACM.

Johnson, E.; Gratch, J.; and DeVault, D. 2017. Towards an autonomous agent that provides automated feedback on students' negotiation skills. In Proceedings of the 16th Conference on Autonomous Agents and MultiAgent Systems, AAMAS '17, 410-418. Richland, SC: International Foundation for Autonomous Agents and Multiagent Systems.

Kelley, H. H. 1996. A classroom study of the dilemmas in interpersonal negotiations. Berkeley Institute of International Studies.

Kim, J. M.; Hill Jr, R. W.; Durlach, P. J.; Lane, H. C.; Forbell, E.; Core, M.; Marsella, S.; Pynadath, D.; and Hart, J.
2009. Bilat: A game-based environment for practicing negotiation in a cultural context. International Journal of Artificial Intelligence in Education 19(3):289-308.

Kolb, A. Y., and Kolb, D. A. 2012. Experiential learning theory. In Encyclopedia of the Sciences of Learning. Springer. 1215-1219.

Lin, R.; Oshrat, Y; and Kraus, S. 2009. Investigating the benefits of automated negotiations in enhancing people's negotiation skills. In Proceedings of The 8th International Conference on Autonomous Agents and Multiagent Systems-Volume 1, 345-352. International Foundation for Autonomous Agents and Multiagent Systems.

Mell, J., and Gratch, J. 2016. Iago: interactive arbitration guide online. In Proceedings of the 2016 International Conference on Autonomous Agents \& Multiagent Systems, 1510-1512. International Foundation for Autonomous Agents and Multiagent Systems.

Monahan, S.; Johnson, E.; Lucas, G.; Finch, J.; and Gratch, J. 2018. Autonomous agent that provides automated feedback improves negotiation skills. In International Conference on Artificial Intelligence in Education, 225-229. Springer. 